\section{Thiyl Radical Catalyzed Enantioselective Cyclizations}

Key words

rational catalyst design

thiyl radical

radical cyclization

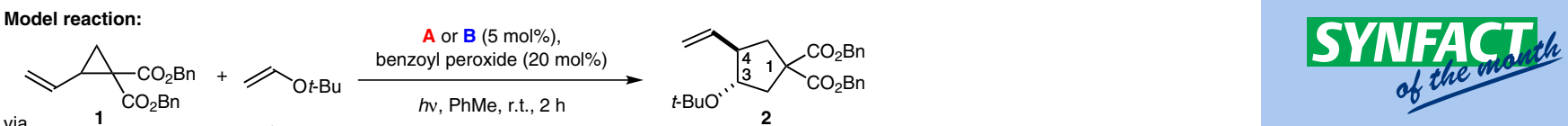

Significance: Maruoka and co-workers report an enantioselective radical cyclization catalyzed by the newly designed thiyl radical catalyst $\mathbf{B}$. The reaction starts from acceptor-substituted vinyl cyclopropanes $\mathbf{1}$, which are opened by the catalyst and reclose to cyclopentane $\mathbf{2}$ after addition to a vinyl ether. Based on the low selectivity obtained with catalyst $\mathbf{A}$ and the transition state model with two quadrants available for the formation of opposite enantiomers, catalyst $\mathbf{B}$ was designed blocking of the quadrants and enabling high enantioselectivities.
Comment: Through rational catalyst design, the Maruoka group has achieved the first highly stereoselective $\mathrm{C}-\mathrm{C}$ bond formation using a thiyl radical catalyst. This proof of principle is expected to spur further research towards the application of catalysts similar to $\mathbf{B}$ and the development of novel thiyl radical catalysts with innovative chiral backbones. Further research is also expected to address the currently poor diastereocontrol between a possible stereocenter at C1 and the anticonfigured stereodiad at C3 and C4. 